\title{
A NEW ENDOBRONCHIAL TUBE FOR LEFT LUNG ANAESTHESIA, WITH SPECIFIC REFERENCE TO RECONSTRUCTIVE PULMONARY SURGERY
}

\author{
BY \\ W. K. PALLISTER \\ From the Brompton Hospital, London
}

(RECEIVED FOR PUBliCATION SEPTEMBER 28, 1958)

Present-day practice in thoracic surgery is to preserve as much healthy lung as is compatible with adequate removal of the disease. Such procedures often involve excision of a diseased segment of main bronchus, and subsequent endto-end anastomosis. When there is distal healthy lung, old healed tuberculous bronchostenosis has been treated in this way (Jackson, Jones, and Holmes Sellors, 1955). Similarly, lobectomy with resection of a "sleeve" of main bronchus has been used in removing advanced upper lobe bronchial carcinoma (Price Thomas, 1956; Johnston and Jones, 1959). This operation is performed on patients in whom poor respiratory reserve precludes pneumonectomy, but in whom the spread of disease to involve the adjacent main bronchus precludes a standard upper lobectomy. The diseased lobe is removed, together with the segment of main bronchus adjoining the upper lobe bronchus. On the left continuity is then restored between the divided main bronchus and the lower lobe bronchus. On the right the lower lobe bronchus, or the intermediate bronchus, is anastomosed to the trachea.

The bronchus must be open for periods of half an hour or more, and smooth, quiet anaesthesia must be maintained during this time. Such conditions are best provided by ventilating the healthy lung through an endobronchial tube positioned in the main bronchus.

The standard Machray left endobronchial tube at present in use has two disadvantages that become more obvious when it is used during sleeve resection of the right upper lobe.

(1) The cuff may burst. While this may be of little importance during tracheal anaesthesia, a burst cuff during endobronchial anaesthesia results in a serious gas leak from the trachea and open bronchus. The concentration of nitrous oxide cannot be maintained and anaesthesia rapidly lightens. Controlled ventilation can no longer be efficiently performed; and spontaneous respiratory effort and mediastinal "flap" may occur. This is harmful to the patient and makes it impossible for the surgeon to proceed.

During sleeve lobectomy the risk of a burst cuff is increased. The upper lobe is often consolidated, or may be hyperinflated with air trapped beyond the growth. Manipulation of the bulky lobe by the surgeon is likely to dislodge the tube upwards, so that the cuff may eventually lie near the carina. It is then liable to be pricked and burst during the anastomosis of the bronchus to the trachea. It is difficult, and dangerous, to attempt to replace the tube while the thorax is open and the patient is in the lateral position.

If, however, a tube is used that has two bronchial cuffs, one lying inside the other, a burst cuff is of no importance. The outer cuff is used first. If this is burst, the inner cuff is inflated and the ventilatory system maintained. This is a simple solution to a problem that is particularly serious when it arises during a reconstructive operation on the bronchus.

(2) Only one lung can be ventilated when a standard endobronchial tube is used. To allow the bronchial suture line to be tested, a single cuffed tube must be drawn back into the trachea. If there is a significant leak, the surgeon must then complete the anastomosis with the operative field obscured by blood-stained froth, for the tube cannot be repositioned in the left main bronchus. It is therefore of value if one or both lungs can be ventilated as required, without displacing the tube. This has been made possible by the addition of a tracheal cuff $3 \mathrm{~cm}$. above the bronchial cuff. Provided the tube is not a "plug" fit in the bronchus, one-lung anaesthesia may be rapidly changed to tracheal anaesthesia by deflating the bronchial cuff and inflating the tracheal cuff. 


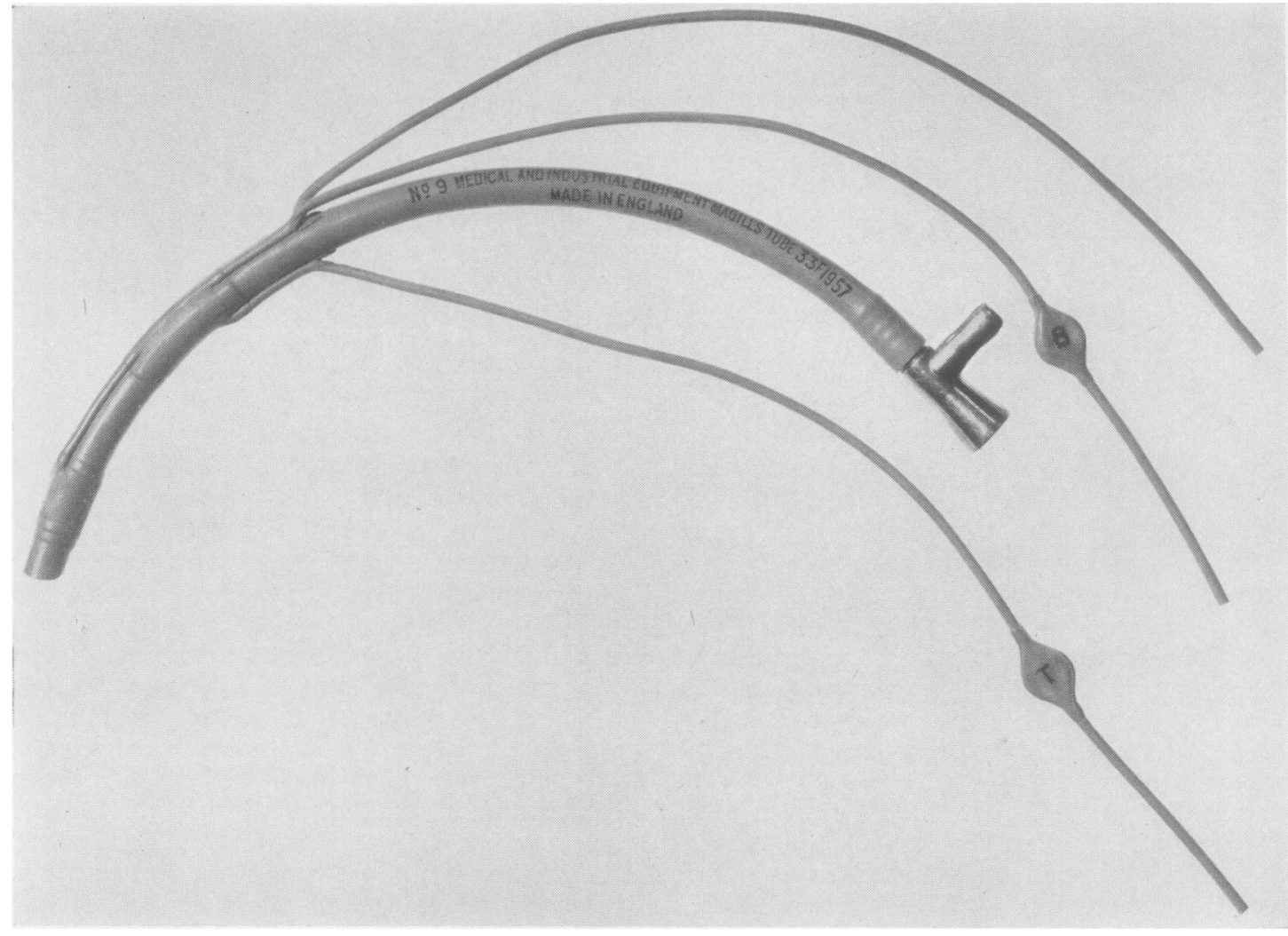

Fig. 1.-The endobronchial tube.

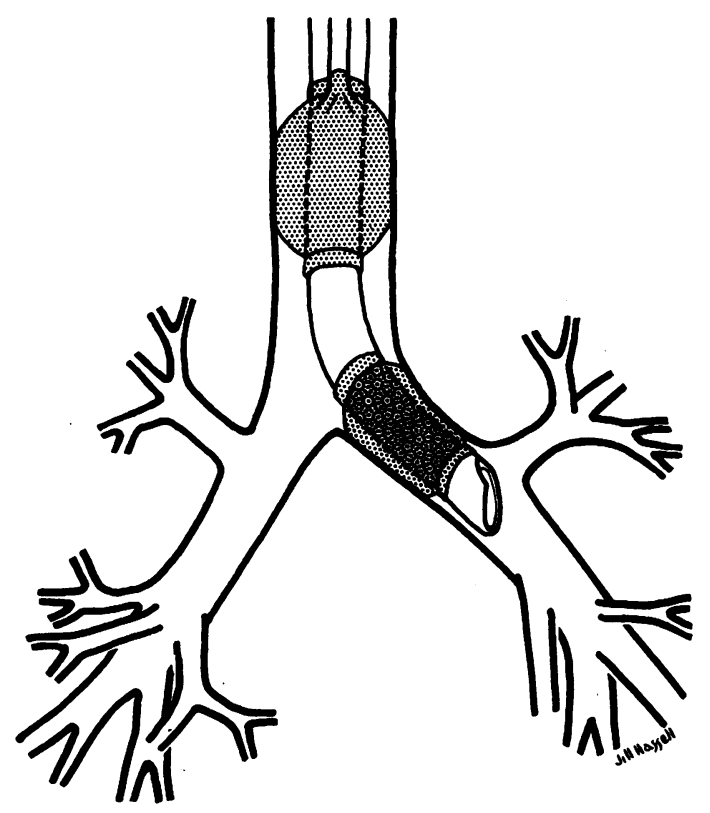

Fig. 2.-Diagrammatic representation of the endobronchial tube in position in the left main bronchus. The distal bronchial cuffs are represented by different stippling patterns. The pilot balloons and inflation tubes are not shown.
Both lungs are now ventilated. It is best to use a No. 8 tube, as a larger tube is likely to be a "plug" fit in the bronchus.

The bronchial cuffs, one inside the other, are $2 \mathrm{~cm}$. long. The tracheal cuff is $4 \mathrm{~cm}$. long, and lies $3 \mathrm{~cm}$. above the distal cuffs. The tube is only slightly more bulky than the single cuffed endobronchial tube.

The outer bronchial cuff has a pilot balloon marked with a B. The inner bronchial cuff has no pilot balloon. The tracheal cuff has a pilot balloon marked $T$. The inflation tubes of the bronchial cuffs are closely parallel on the posterior aspect of the tube as it lies in the larynx. The tracheal inflation tube is on the anterior surface of the tube, the major axis of which lies, therefore, anteroposteriorly between the cords.

There may be difficulty in placing a No. 8 endobronchial tube ovet the $8 \mathrm{~mm}$. Magill bronchoscope and in sliding it off when the tube is in position in the bronchus. This is avoided by first heating the tube for a minute in boiling water, to soften it, and then lubricating the outside of the bronchoscope liberally with glycerine. The tube will then slide easily on or off the bronchoscope. 
When passing the tube, care must be taken that the tracheal cuff is not torn against the incisor teeth. The bronchoscope is advanced into the left main bronchus until the orifice of the upper lobe bronchus is seen. It is then withdrawn from the tube and the outer bronchial and tracheal cuffs inflated. This stabilizes the tube while the patient is positioned. The head should be extended to avoid the tube kinking in the oropharynx. The bronchial cuff should now lie in the left main bronchus, without risk of obstructing either the left upper lobe bronchus or the right main bronchus.

Normally the harmful effects of a pulmonary vascular shunt through unventilated lung are most noticeable while the chest is being opened and before the lung has been allowed to deflate. By releasing the bronchial cuff, both lungs can be ventilated until the chest is open and a shunt thus prevented. One-lung anaesthesia is then re-established by inflation of the bronchial cuff. In the presence of copious secretion it is, of course, unsafe to use the tube other than to provide one-lung anaesthesia.

Only the bronchial cuff is left inflated once the chest is open. This allows the diseased lung to collapse and the secretions expressed by the surgeon's manipulation to drain freely via the trachea to the oropharynx, whence they are removed by suction.

For left upper lobectomy with sleeve resection the Gordon-Green right endobronchial tube was first used. Later, a modification of this tube, which can be passed visually on a Magill bronchoscope, proved of value. The tube has bronchial and tracheal cuffs and is used in a similar manner to that described for the left endobronchial tube. It allows ventilation of the right lung, or of both lungs together. The bronchial anastomosis can thus be tested without withdrawing the tube into the trachea.

Both the new left endobronchial tube described and the modified Gordon-Green tube are introduced on the Magill bronchoscope, which is then withdrawn to leave the tube in position (Magill, 1936 ; Mansfield, 1957). " Blind " methods of positioning endobronchial tubes are neither safe nor reliable. When the bronchial anatomy is normal a tube passed blindly will almost invariably enter the right main bronchus. In the presence of old tuberculous disease, or an upper lobe neoplasm, the bronchi are often so distorted that the position of a tube passed in this way is completely uncertain. It is likely to be traumatic and bleeding may occur. In many patients there may be copious pus, as, for example, from a carcinomatous abscess, which may flood the opposite lung on the induction of anaesthesia. Bronchoscopic intubation is, therefore, considered to be the only safe method.

The endobronchial tube described has been in use for over two years. It has solved the mechanical problems of ventilation during anaesthesia for right sleeve resection and has proved useful for other operative procedures in which left lung anaesthesia has been required.

\section{SUMMARY}

The mechanical problems of ventilation during left endobronchial anaesthesia are discussed, with particular reference to those arising during right upper lobectomy with resection and anastomosis of the main bronchus. The advantages of a new endobronchial tube are described and its method of use discussed. Reference is made to the similar method of using a modified Gordon-Green tube during left sleeve resection. The need for visual intubation is stressed as being the only accurate and safe method.

I am indebted to Sir Clement Price Thomas, whose work on sleeve resection stimulated the development and clinical application of the tube; and to the consultant surgeons and anaesthetists of the Brompton Hospital for their help and encouragement.

I wish to thank Mr. D. Kemp, of the Photographic Department of the Institute of Diseases of the Chest, for the photograph of the tube, and also Miss Jill Hassell, medical artist of the Department of Medical Photography, Westminster Hospital, for the drawing of the tube positioned in the bronchial tree.

I am grateful to Mr. Childerhouse of Medical Industrial Equipment Ltd., who made the prototype tubes, and to Miss P. Dawson for typing the manuscript.

\section{REFERENCES}

Jackson, J. W., Jones, P. H., and Holmes Sellors, T. (1955). Thorax, 10, 229.

Johnston, J. B., and Jones, P. H. (1959). Ibid., 14, 48.

Magill, I. W. (1936). Proc. roy. Soc. Med., $29,643$.

Mansfield, R. E. (1957). Anaesthesia, 12, 477.

Thomas, Sir C. Price (1956). J. roy. Coll. Surg. Edinb., 1, 169. 